\title{
Macrophages, fat, and the emergence of immunometabolism
}

\author{
Anthony W. Ferrante Jr. \\ Department of Medicine, Naomi Berrie Diabetes Center, Columbia University, New York, New York, USA.
}

\begin{abstract}
Over the last century, our modern concepts and understanding of metabolism and immunology have evolved largely in parallel. Notably, during the last decade, there has been a sharpened focus on the convergence of metabolism and immune function. In part motivated by studies originally published in the JCI, we now recognize that the immune system monitors the metabolic state of tissues and organisms and responds in kind by modulating metabolic function. The complexity of these interactions, both adaptive and pathologic, continues to be studied and revealed, with the hope that harnessing the reins that control immune function may one day be used for metabolic benefit.
\end{abstract}

\section{Inflammatory pathways implicated in metabolic disease}

For more than 50 years, physicians and scientists have observed a tight association between metabolic disorders and systemic inflammation. Studies dating from the 1960s found that individuals with what we now recognize as type 2 diabetes mellitus have higher circulating concentrations of active complement and acute phase reactants $(1,2)$, both of which are classic markers of an inflammatory state. Inflammation is a broad term applied to a variety of processes, but most often indicates stress to an underlying system. In the context of metabolic disease, the site of inflammatory stress and the physiologic and pathologic implications of the inflammatory source remained obscure until about 20 years ago.

At that time, Gokhan Hotamisligil, working in Bruce Spiegelman's laboratory, found that obesity induced expression of the prototypical inflammatory protein, TNF- $\alpha$, in adipose tissue of both rodents and humans. Additionally, Hotamisligil and Spiegelman determined that measurable increases in circulating TNF- $\alpha$ were attributable to adipose tissue and postulated that TNF- $\alpha$ mediates obesity-associated insulin resistance (3). While the precise role of TNF- $\alpha$ in mediating insulin resistance and type 2 diabetes remains incompletely defined, other groups have built on these

Conflict of interest: The author has declared that no conflict of interest exists.

Citation for this article: J Clin Invest. 2013;123(12):4992-4993. doi:10.1172/JCI73658. initial observations to demonstrate that obesity activates the expression of more than 50 inflammatory molecules in fat (4).

\section{The correlation between macrophages and adiposity}

Ten years ago Hong Chen, then leading a group at Millenium Pharmaceuticals, and our laboratory separately studied expression profiles in adipose tissue of mice. We both surprisingly found transcriptional evidence for the presence of macrophages $(5,6)$. In our study, we profiled adipose tissue from mice that varied widely in adiposity due to differences in sex, diet, and segregation of alleles that cause genetic forms of obesity. Because adiposity is a quantitative trait that correlates with medically important quantitative phenotypes, we reasoned that transcriptional profiling might reveal adipose tissue alterations that varied with mass. In what seems to be a relatively simplistic approach by current standards, we asked whether we could identify genes whose expression correlated with measures of adiposity. We identified more than 1,300 such transcripts and I, along with MD-PhD student Stuart Weisberg, began to pore over them.

As a graduate student, most of my efforts were directed at identifying genes involved in Drosophila development. However, these studies were done with E. Richard Stanley, whose laboratory is devoted to studying macrophage development. As a consequence, I sat through many journal clubs and lab meetings during which mouse macrophage-specific genes and proteins were discussed. As Stuart and I looked down the list, it became apparent that many of the genes that positively correlated with adiposity in adipose tissue were familiar to me from my time in the Stanley laboratory. I called Richard and asked whether he could provide me with antibodies and some macrophage-deficient mice to test whether the gene signatures were indeed reflective of the macrophage content of the adipose tissue. The immunohistochemistry was quite striking. Though we were originally doubtful that such a simple explanation could explain our expression data, we were quickly convinced that typical rodent and human adipose tissues contain macrophages at levels proportionate to adiposity. We also demonstrated that most of the TNF- $\alpha$ and several other classical inflammatory molecules were derived from adipose tissue macrophages.

\section{The intersection of metabolic dysfunction and inflammation}

These initial observations provided a new context for understanding obesity-induced inflammation (7). What had previously been thought to be the isolated release of inflammatory molecules by adipocytes in response to hypertrophy now clearly represented a complex conversation between stressed adipocytes and the immune system. In the intervening years, work by many laboratories has revealed that obesity induces a complex and broad immune response involving nearly all innate and adaptive immune cell types. The initiating immune event or events remain unclear; some studies suggest infiltration of neutrophils (8), others implicate reductions in regulator T cells (9), and others propose rapid changes in macrophages (10). Nonetheless, it is clear that metabolic-induced changes in immune cell number and function are coordinated and profound (11).

Although the initial immunometabolism studies focused on adipose tissue, it is now evident that metabolic activation of the immune system is not limited to fat of obese individuals. Obesity has now been shown to change immune cell 
populations in the liver, bone marrow, key regulator areas of the hypothalamus, and the circulation. Furthermore, metabolic regulation of the immune system is not limited to obesity. Fasting, cold challenge, weight loss, and acute lipolysis all increase the macrophage number and function in adipose tissue. Perhaps this should not have been surprising given the inhibitory effects of starvation and reduced leptin concentrations on immune function and effector $\mathrm{T}$ cells. The emerging narrative argues that the immune system monitors and responds to the metabolic state of tissues and the whole organism.

Building on earlier work that indicates TNF- $\alpha$ mediates obesity-associated insulin resistance and identifies a role for immune cells in atheromas as well as a great deal of evidence provided by rodent studies, a model in which the immune system contributes directly to the development of obesity-induced pathologies, including type 2 diabetes, nonalcoholic fatty liver disease, and, of course, atherosclerosis has emerged $(12,13)$. Although at least one broad classical antiinflammatory agent (salsalate) improved glycemic control in a randomize trial in patients with type 2 diabetes mellitus, it appears to have done so without reducing insulin resistance as predicted by the current models (14). Furthermore, despite some encouraging results from small trials (15), the lack of consistent therapeutic success in targeting specific immune molecules to treat metabolic disease has provided some frustration (16). The relative paucity of immune-related genes uncovered in genome-wide association studies of obesity-related phenotypes in humans (17) suggests the simple elegance of immune dysfunction leading to metabolic disease indicated by rodent models may be misleading. We are awaiting the results of several large-scale clinical trials.

Much of the research characterizing the interaction of the immune and metabolic systems has focused on maladaptive immune responses; very little is known about adaptive homeostatic functions in metabolism. A compelling argument can be made that obesity is an evolutionary aberration that was not previously experienced by a wide swath of any population; therefore, the immune response to this recent stress reflects uncharacteristic biological clumsiness, resulting in insulin resistance, hepatic steatosis, and $\beta$ cell failure. However, I suspect underlying adaptive functions of the immune system's response to obesity will be found. The mammalian immune system has evolved to restore local homeostasis to tissues under endogenous and exogenous stress, and recent work on cold challenge in mice and weight loss argues that there are evolutionarily ancient metabolic states that the immune system recognizes and responds to adaptively.

\section{The future of immunometabolism}

Although its roots run long and deep in the scientific literature, immunometabolism is a young field with many questions to be answered. In the past decade, the metabolic and, increasingly, the immunology communities have succeeded in identifying key players and providing fundamental descriptions of basic immunometabolic processes. In the coming decade, I imagine that we will uncover and further flesh out adaptive roles of the immune system in metabolism. A more detailed understanding of immunometabolism will permit more focused immune therapies to target metabolic diseases, and a greater appreciation for the ability of metabolic systems to modulate classic immune function will enhance our understanding of autoimmune and inflammatory disorders.

\section{Acknowledgments}

This work was supported by grants from the National Institute of Diabetes and Digestive and Kidney Diseases (NIDDK) (DK066525 and DK063608) and the Lewis Katz Foundation.
Address correspondence to: Anthony W. Ferrante Jr., Department of Medicine, Naomi Berrie Diabetes Center, Columbia University, New York, New York 10032, USA. Phone: 212.851.5322; Fax: 212.851.5335; E-mail: awf7@columbia.edu.

1. Ganrot PO, Gydell K, Ekelund H. Serum concentration of $\alpha$-2-macroglobulin, haptoglobin and $\alpha$-1-antitrypsin in diabetes mellitus. Acta Endocrinol (Copenh). 1967;55(3):537-544.

2. Powell ED, Field RA. Studies on salicylates and complement in diabetes. Diabetes. 1966;15(10):730-733.

3. Hotamisligil GS, Shargill NS, Spiegelman BM. Adipose expression of tumor necrosis factor- $\alpha$ : direct role in obesity-linked insulin resistance. Science. 1993;259(5091):87-91.

4. Gregor MF, Hotamisligil GS. Inflammatory mechanisms in obesity. Annu Rev Immunol. 2011; 29:415-445.

5. Weisberg SP, McCann D, Desai M, Rosenbaum M, Leibel RL, Ferrante AW Jr. Obesity is associated with macrophage accumulation in adipose tissue. J Clin Invest. 2003;112(12):1796-1808.

6. Xu H, et al. Chronic inflammation in fat plays a crucial role in the development of obesity-related insulin resistance. J Clin Invest. 2003;112(12):1821-1830.

7. Wellen KE, Hotamisligil GS. Obesity-induced inflammatory changes in adipose tissue. $\mathrm{J}$ Clin Invest. 2003;112(12):1785-1788.

8. Talukdar S, et al. Neutrophils mediate insulin resistance in mice fed a high-fat diet through secreted elastase. Nat Med. 2012;18(9):1407-1412.

9. Feuerer M, et al. Lean, but not obese, fat is enriched for a unique population of regulatory $\mathrm{T}$ cells that affect metabolic parameters. Nat Med. 2009; 15(8):930-939.

10. Weisberg SP, et al. CCR2 modulates inflammatory and metabolic effects of high-fat feeding. J Clin Invest. 2006;116(1):115-124.

11. Odegaard JI, Chawla A. The immune system as a sensor of the metabolic state. Immunity. 2013; 38(4):644-654

12. Donath MY, Shoelson SE. Type 2 diabetes as an inflammatory disease. Nat Rev Immunol. 2011; 11(2):98-107.

13. Osborn O, Olefsky JM. The cellular and signaling networks linking the immune system and metabolism in disease. Nat Med. 2012;18(3):363-374.

14. Goldfine AB, et al. Salicylate (salsalate) in patients with type 2 diabetes: a randomized trial. Ann Intern Med. 2013;159(1):1-12.

15. Larsen CM, et al. Interleukin-1-receptor antagonist in type 2 diabetes mellitus. $N$ Engl J Med. 2007;356(15):1517-1526.

16. Bernstein LE, Berry J, Kim S, Canavan B, Grinspoon SK. Effects of etanercept in patients with the metabolic syndrome. Arch Intern Med. 2006;166(8):902-908.

17. Manning AK, et al. A genome-wide approach accounting for body mass index identifies genetic variants influencing fasting glycemic traits and insulin resistance. Nat Genet. 2012;44(6):659-669. 